\title{
Stability of Dynamic Signatures: From the Representation to the Generation Domain
}

\author{
Giuseppe Pirlo ${ }^{1}$, Donato Impedovo ${ }^{1}$, Rejean Plamondon ${ }^{2}$, Christian O'Reilly ${ }^{2}$, \\ A. Cozzolongo ${ }^{1}$, R. Gravinese ${ }^{1}$, and Andrea Rollo ${ }^{1}$ \\ ${ }^{1}$ Dipartimento di Informatica, Università degli Studi di Bari, Italy \\ ${ }^{2}$ Ecole Polytecnique de Montreal, Canada \\ giuseppe.pirlo@uniba.it
}

\begin{abstract}
Stability analysis of handwritten signatures is very relevant for automatic signature verification. In this paper the analysis of stability is performed by considering the characteristics of the processes underlying signature generation. More precisely, the analysis of stability is performed by considering the Sigma-Lognormal parameters, according to the Kinematic Theory. The experimental tests, carried out using the SUSig database, demonstrate that the new technique can provide useful information both for a deep understanding of the processes of signature generation and for the improvement of the processes for automatic signature verification.
\end{abstract}

Keywords: Biometry, Automatic Signature Verification, Stability Analysis, Sigma-Lognormal.

\section{Introduction}

In the contemporary society, handwritten signature is a common trait for personal authentication and people are familiar with its use [1].

Unfortunately, a handwritten signature is a very complex trait. The rapid writing movement underlying handwritten signature generation is determined by a motor program stored into the signer's brain and implemented through the signer's writing system and writing devices (paper and pen type, etc.). Therefore, each handwritten signature strongly depends on a multitude of factors such as the psychophysical state of the signer and its social and cultural environment as well as the conditions under which the signature apposition acquisition process occurs [2,3]. Hence, it is not surprising that although there is a growing interest in the field of automatic signature verification, several basic aspects concerning the nature of this very special kind of biometric trait are still open to investigation. Indeed, research on automatic signature verification is a multifaceted field of research involving aspects from disciplines as wide ranging as human anatomy and engineering, neuroscience and computer sciences, and so on.

One of the basic aspects that is currently at the centre of a large debate concerns signature stability [4,5]. In fact, everyone is aware that his/her signature is never the 
same even if each of us generally learns to sign at an early age and practices constantly to produce similar signatures according to his/her specific and personal model. Hence, stability in handwritten signatures is a crucial characteristic for investigating the intrinsic human properties related to handwriting generation processes concerning human psychology and biophysics. In addition, its study can provide new insights for a more accurate treatment of signatures for verification purposes, hence contributing to the design of more effective signature verification systems. For these reasons, it is not surprising that the scientific community has been devoting much effort to the analysis of signature stability.

When considering dynamic signatures, many approaches estimate signature stability by the analysis of a specific set of characteristics. In general, these approaches have shown that there is a set of features which remain stable over long time periods, while others can change significantly in time [6,7]. More precisely, a comparative study of the consistency of certain features of dynamic signatures has demonstrated that position, velocity and pen inclination can be considered to be among the most consistent, when a distance-based consistency model is applied [6]. Other results based on personal entropy demonstrated that position is a stronger characteristic than pressure and pen inclination in both short and long-term variability. Moreover, although pressure may give better performance results in a short-term context, it is not recommended for signature verification in a long-term.

When static signatures are considered, the degree of stability of each region of a signature can be estimated by a multiple pattern-matching technique [8,9]. The basic idea is to match corresponding regions of genuine signatures in order to estimate the extent to which they are locally different. Of course, a preliminary step is used to determine the best alignment of the corresponding regions of signatures, in order to diminish any differences among them.

This paper presents a new technique for the analysis of local stability in dynamic signature. This technique applies Dynamic Time Warping (DTW) to match signatures in their generation domain according to the Sigma-Lognormal model. The organization of this paper is the following. Section 2 presents the Sigma- Lognormal model. Section 3 presents the new technique for the analysis of stability based on Dynamic Time Warping and Section 4 presents the experimental results, carried out on signatures of the SuSig database. Finally, section 5 presents the conclusion of the work.

\section{Sigma-Lognormal Model}

The kinematic theory of rapid human movement[10], relies on the Sigma-Lognormal model to represent the information of both the motor commands and timing properties of the neuromuscular system involved in the production of complex movements like signature [11,12].

The Sigma-Lognormal model considers the resulting speed of a single stroke $j$ as having a lognormal shape $\Lambda$ scaled by a command parameter (D) and time-shifted by the time occurrence of the command ( $\mathrm{t} 0)$ : 


$$
\left|\vec{v}_{j}\left(t ; P_{j}\right)\right|=D_{j} \Lambda\left(t-t_{0 j} ; \mu_{j}, \sigma_{j}^{2}\right)=\frac{D_{j}}{\sigma\left(t-t_{0 j}\right) \sqrt{2 \pi}} \exp \left\{\frac{\left[\ln \left(t-t_{0 j}\right)-\mu_{j}\right]^{2}}{-2 \sigma_{j}^{2}}\right\}
$$

where $\operatorname{Pj}=\left[\mathrm{D}_{\mathrm{j}}, \mathrm{t}_{0 \mathrm{j}}, \mu_{\mathrm{j}}, \sigma_{\mathrm{j}}, \Theta_{\mathrm{sj}}, \Theta_{\mathrm{ej}}\right]$ represents the sets of Sigma-Lognormal parameters[11]:

- $\mathrm{D}_{\mathrm{j}}$ : amplitude of the input commands;

- $\mathrm{t}_{0 \mathrm{j}}$ : time occurrence of the input commands, a time-shift parameter;

- $\mu_{\mathrm{j}}$ : log-time delays, the time delay of the neuromuscular system expressed on a logarithmic time scale;

- $\sigma_{\mathrm{j}}$ : log-response times, which are the response times of the neuromuscular system expressed on a logarithmic time scale;

- $\Theta_{\mathrm{sj}}$ : starting angle of the circular trajectories described by the lognormal model along pivot;

- $\Theta_{\mathrm{ej}}$ : ending angle of the circular trajectories described by the lognormal model along pivot.

Additionally, from the hypothesis that every lognormal stroke represents the movement as happening along pivot, the angular position can be computed as [11]:

$$
\phi_{j}\left(t ; P_{j}\right)=\theta_{s j}+\frac{\theta_{e j}-\theta_{s j}}{D_{j}} \int_{0}^{t}\left|\vec{v}\left(\tau ; P_{j}\right)\right| d \tau
$$

In this context, a signature can be seen as the output of a generator that produces a set of individual strokes superimposed in time. The resulting complex trajectories can be modeled as a vector summation of lognormal distributions (being $\mathrm{N}_{\mathrm{LN}}$ the total number of lognormal curves in which the handwritten trace is decomposed):

$$
\vec{v}(t)=\sum \Lambda(t)=\sum_{j=1}^{N_{L N}} \vec{v}_{j}\left(t ; P_{j}\right)
$$

For each of the components of the signature, and then for each stroke, it can define some profiles that add information to those already expressed by the parameters of the Sigma-Lognormal [10]. For the aims of this paper, the most relevant profile is the velocity profile, that is expressed by this expression:

$$
v_{i}(t)=\frac{D_{i}}{\sigma_{i}\left(t-t_{0 i} \sqrt{2 \pi}\right)} e^{-\frac{1}{2 \sigma_{i}^{2}\left[\ln \left(\left(t-t_{0 i}\right)\right)-\mu_{i}\right]^{2}}}
$$

Of course, expression (4) reaches its maximum for 


$$
t=t_{0}+\exp \left(-\sigma^{2}+\mu\right)
$$

According to the Sigma-Lognormal model, in this paper a signature $S^{t}$ is characterized in the generation domain by a sequence of couples

$$
S^{r}=\left(z_{1}^{r}, z_{2}^{r}, z_{3}^{r}, \ldots z_{j}^{r}, \ldots, z_{m}^{r}\right)
$$

where each couple $z_{j}^{r}=\left(t_{j}, v_{j}\left(t_{j}\right)\right)$ describes synthetically the $j$-th lognormal curve in which the signature is decomposed (in eq. (6) it is supposed that a signature is decomposed in m lognormal curves).

\section{$3 \quad$ Analysis of Stability by Dynamic Time Warping}

Stability analysis is here performed by the DTW, that is used to match the sequences representing sets of genuine signatures. Precisely, a local stability function is here defined starting from a set $S=\left\{S^{r} \mid r=1,2, \ldots, n\right\}$ of $n$ hand-written signatures of the same writer. According to eq. (6) $S^{r}$ is described as [13]:

$$
S^{r}=\left(z_{1}^{r}, z_{2}^{r}, z_{3}^{r}, \ldots z_{j}^{r}, \ldots, z_{m}^{r}\right)
$$

Let be $S^{r}, S^{t}$ two signatures of $S$. A warping function between $S^{r}$ and $S^{t}$ is any sequence of couples of indexes identifying points of $S^{r}$ and $S^{t}$ to be joined:

$$
\mathrm{W}\left(\mathrm{S}^{\mathrm{r}}, \mathrm{S}^{\mathrm{t}}\right)=\mathrm{C}^{1}, \mathrm{C}^{2}, \ldots \mathrm{C}^{\mathrm{k}}
$$

where $c^{\mathrm{k}}=\left(\mathrm{i}^{\mathrm{k}}, \mathrm{j}^{\mathrm{k}}\right) \quad\left(\mathrm{k}, \mathrm{i}^{\mathrm{k}}, \mathrm{j}^{\mathrm{k}}\right.$ integers, $\left.1 \leq \mathrm{k} \leq \mathrm{K}, 1 \leq \mathrm{i}^{\mathrm{k}} \leq \mathrm{M}^{\mathrm{r}}, 1 \leq \mathrm{j}^{\mathrm{k}} \leq \mathrm{M}^{\mathrm{t}}\right)$.

Now, if we consider a distance measure

$$
\mathrm{D}\left(\mathrm{C}^{\mathrm{k}}\right)=\mathrm{d}\left(\mathrm{z}_{\mathrm{i}}^{\mathrm{r}} \mathrm{k}, \mathrm{z}_{\mathrm{j}}^{\mathrm{t}}{ }\right)
$$

between points of $S^{r}$ and $S^{t}$, we can associate to warping function in (9) the dissimilarity measure

$$
D\left(\pi\left(S^{\prime}, S^{\prime}\right)\right)=\sum_{j=1}^{\pi} d\left(C^{i}\right)
$$

The elastic matching procedure detects the warping function

$$
\mathrm{W}^{*}\left(\mathrm{~S}^{\mathrm{r}}, \mathrm{S}^{\mathrm{t}}\right)=\mathrm{C}^{* 1}, \mathrm{C}^{* 2}, \ldots, \mathrm{C}^{* \mathrm{~K}}
$$

which satisfies the monotonicity, continuity and boundary conditions, and for which it results [4] 


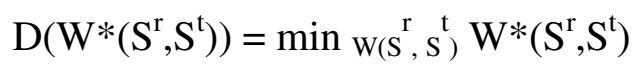

From the warping function in (11) we identify the Direct Matching Points (DMP) of $S^{r}$ with respect to $S^{t}[4,14]$.

In the generation domain, a DMP of a signature $S^{r}$ with respect to $S^{t}$ is a lognormal curve which has a one-to-one coupling with a lognormal curve $S^{t}$. In other words, let $\mathrm{z}_{\mathrm{p}}^{\mathrm{r}}$ be a curve of $\mathrm{S}^{\mathrm{r}}$ coupled with $\mathrm{z}_{\mathrm{q}}^{\mathrm{t}}$ of $\mathrm{S}^{\mathrm{t}} ; \mathrm{z}^{\mathrm{r}}(\mathrm{p})$ is DMP of $\mathrm{S}^{\mathrm{r}}$ with respect to $S^{t}$ iff $[14,15,16]$ :

- $\forall \mathrm{p}^{\prime}=1, \ldots, \mathrm{M}^{\mathrm{r}}$, it results that $\mathrm{z}_{\mathrm{p}}^{\mathrm{r}}$, is not coupled with $\mathrm{z}_{\mathrm{q}}^{\mathrm{t}}$;

- $\forall \mathrm{q}^{\prime}=1, \ldots, \mathrm{M}^{\mathrm{t}}$, it results that $\mathrm{z}_{\mathrm{q}^{\mathrm{t}}}^{\mathrm{t}^{\prime}}$ is not coupled with $\mathrm{z}_{\mathrm{p}}^{\mathrm{r}}$;

Now, a DMP indicates the existence of a curve of the generation process of the r-th signature which is roughly similar to the corresponding curve of the $t$-th signature. Therefore, for each curve of index $\mathrm{p}$ of $\mathrm{S}^{\mathrm{r}}$, a score is introduced according to its type of coupling with respect to the corresponding index of $S^{t}[17]$ :

- $\operatorname{Score}^{\mathrm{t}}\left(\mathrm{z}_{\mathrm{p}}^{\mathrm{r}}\right)=1$ if $\mathrm{z}_{\mathrm{p}}^{\mathrm{r}}$ is a DMP

- $\operatorname{Score}^{t}\left(z_{p}^{r}\right)=0$ if $z_{p}^{r}$ is not a DMP

Of course, when the genuine signature $\mathrm{S}^{t}$ is matched against all the genuine signatures of the set (7), we can derive the local stability function of $S^{t}$ for each lognormal curve, by averaging the scores obtained from eqs. (14) as follows :

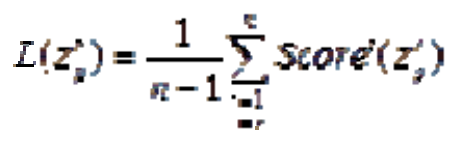

The global stability function for the signature $S^{t}$ can be defined as:

$$
G\left(z^{y}\right)=\frac{1}{m} \sum_{f=:}^{n} L\left(z_{f}\right)
$$

\section{Experimental Results}

For the testing stage the signatures of the Visual SubCorpus of the SuSig database (developed at the Sabançi University - Turkey) were used. The Visual SubCorpus is composed by 10 genuine signatures and 10 forgery signatures, acquired by 94 authors, that are maximally students or associates of the Sabançi University, aged between 21 and 52 years [15]. 


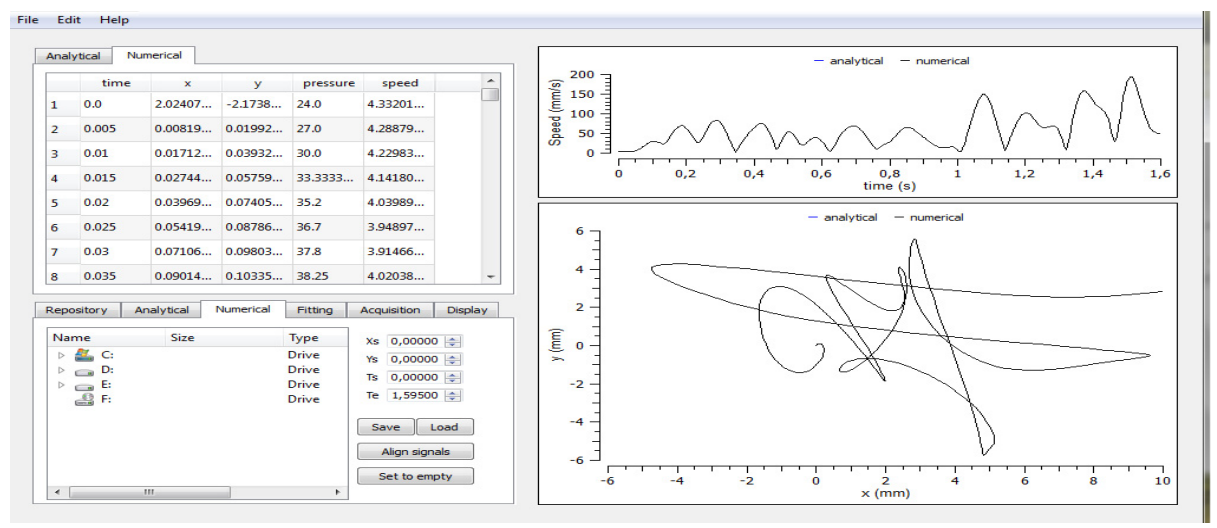

Fig. 1. An example of using ScriptStudio for parameter extraction

For each signature, Sigma-Lognormal parameters were extracted using Script Studio [11]. Figure 1 shows an example of parameter extraction for a signature of the SuSig database.

The parameters are modeled in a matrix, in which in each row contains the parameters of a Sigma-Lognormal curve of the signature, such as: stroke initial time; stroke amplitude; log time delay; log response time; initial angle; final angle.

Figures 2 and 3 show respectively the velocity profiles obtained by the SigmaLognormal curves for a genuine and a forged signature. It is worth noting that the analysis of the signatures in the generation domain (through the lognormal model) allows us to have a first indication of the authenticity of a signature. In fact, the number of curves underlying a forgery signature is generally higher than the number of curves that underlying the corresponding genuine specimen. This can be explained with the presence of additional strokes that are created by the forger in an attempt to imitate particularly complex traits of the genuine signature.
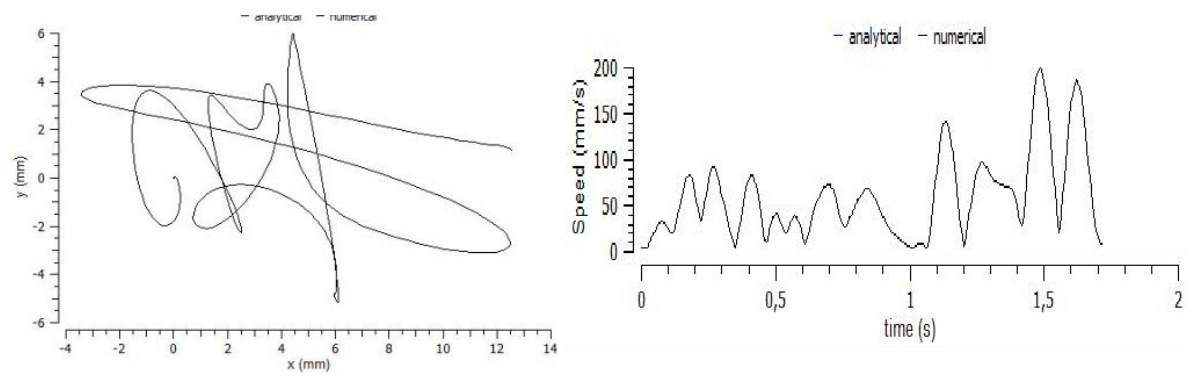

Fig. 2. Example of genuine signature and related velocity profile 

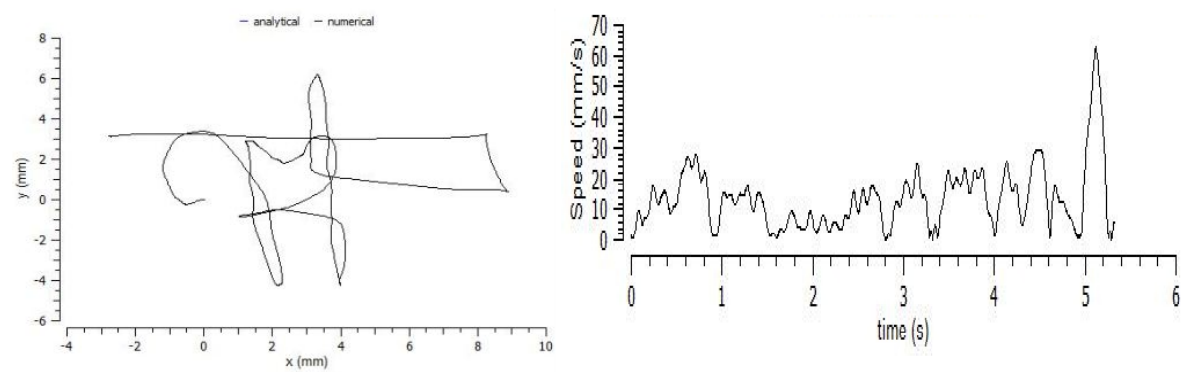

Fig. 3. Example of forgery signature and related velocity profile

For the analysis of local stability of the generation process a well-defined Matlab function was realized with the following purposes:

1. determine time and velocity profile for the target signature, that is first signature produced by author;

2. determine time and velocity profile for signatures that must be matched with the target signature;

3. determine the warping path using Dynamic Time Warping (TDW);

4. count the number of DMP for each stroke;

5. compute the local stability function (according to eq. (15));

6. compute the global stability for each signature (according to eq. (16))

Figure 4 shows the stability function for a genuine signature, when it is matched against other genuine specimens (genuine-genuine) and forgeries (genuine-forgeries).

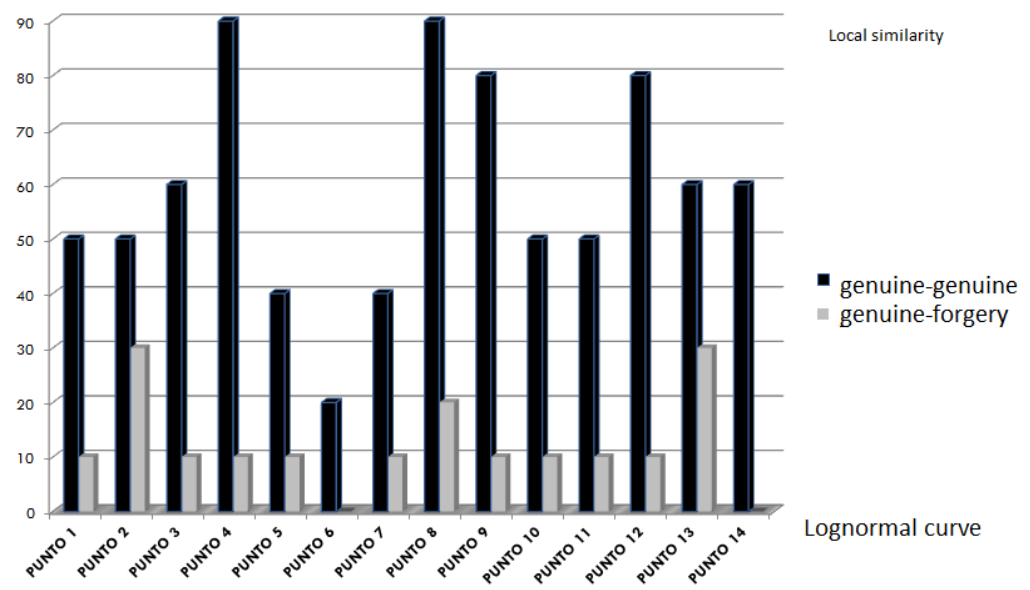

Fig. 4. An example of results obtained by analyzing local stability 
The result shows that generally the local stability among genuine-genuine signatures is significantly greater than local stability among genuine-forgeries. In particular, some curves exist in which stability of genuine signatures is clearly superior to those of the forgeries signature (examples are curves 4 and 8), while for other curves the difference is not so evident (examples are curves 2 and 13).

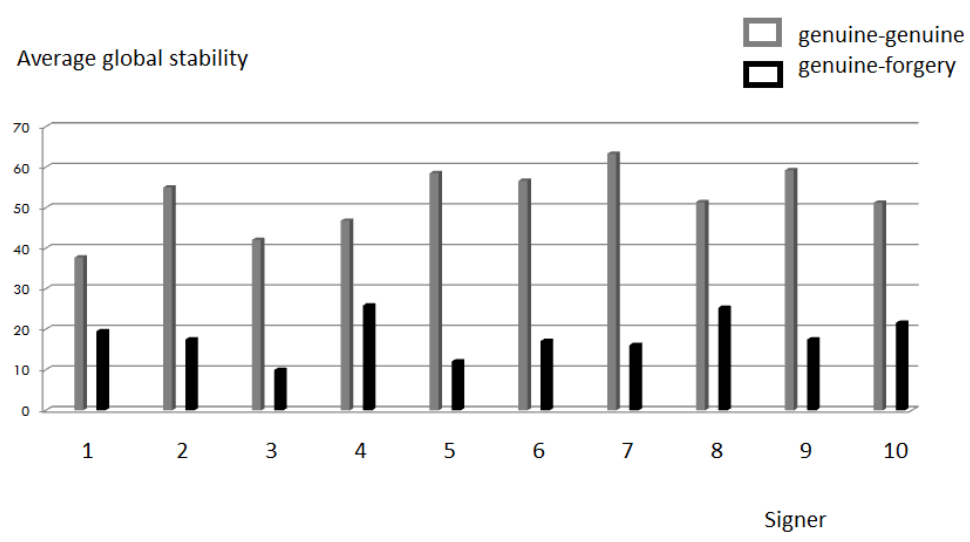

Fig. 5. An example of results obtained by analyzing global stability

As regards the global stability, figure 5 shows the results obtained by 10 authors, taken randomly from the 94 authors of Visual Subcorpus. For each author the averaged value of the global stability function of their signatures is reported, that provide an information about the stability of the signatures of each signer. In this case the result demonstrate that generally global stability of the signatures produced by the signer exceeds $60 \%$, while global stability of the signatures produced by forgeries is just over $20 \%$.

\section{Conclusions}

In this paper, a new approach for the analysis of local and global stability for online signatures was presented. This approach is based on Dynamic Time Warping algorithm applied to signatures modeled by speed profiles extracted from appropriate parameters of Sigma-Lognormal distributions. The experimental results demonstrate that the new approach can provide useful information about signatures that reveal some specific characters about signers' behaviors.

\section{References}

1. Vielhauer, C.: A Behavioural Biometrics. Public Service Review: European Union 9, $113-115$ (2005)

2. Plamondon, R.: A Kinematic Theory of Rapid Human Movements: Part I: Movement Representation and generation. Biological Cybernetics 72(4), 295-307 (1995) 
3. Impedovo, D., Pirlo, G., Plamondon, R.: Handwritten Signature Verification: New Advancements and Open Issues. In: XIII International Conference on Frontiers in Handwriting Recognition (ICFHR 2012), Monopoli, Bari, Italy, pp. 365-370 (September 2012)

4. Huang, K., Yan, H.: Stability and style-variation modeling for on-line signature verification. Pattern Recognition 36(10), 2253-2270 (2003)

5. Impedovo, S., Pirlo, G.: Verification of Handwritten Signatures: an Overview. In: 14th International Conference on Image Analysis and Processing, ICIAP 2007, Modena, Italy, pp. 191-196. IEEE Computer Society Press (September 2007)

6. Lei, H., Govindaraju, V.: A comparative study on the consistency of features in on-line signature verification. Pattern Recognition Letters 26, 2483-2489 (2005)

7. Schomaker, L.R.B., Plamondon, R.: The Relation between Axial Pen Force and Pen Point Kinematics in Handwriting. Biological Cybernetics 63, 277-289 (1990)

8. Impedovo, D., Pirlo, G.: On the Measurement of Local Stability of Handwriting - An application to Static Signature Verification. In: Biometric Measurements and Systems for Security and Medical Applications (BIOMS 2010), Taranto, Italy, pp. 41-44. IEEE Computer Society Press (September 2010)

9. Impedovo, D., Pirlo, G., Stasolla, E., Trullo, C.A.: Learning Local Correspondences for Static Signature Verification. In: 11th Int. Conf. of the Italian Association for Artificial Intelligence (AI*IA 2009), Reggio Emilia, Italy (December 2009)

10. Djioua, M., Plamondon, R.: Studying the Variability of Handwriting Patterns using the Kinematic Theory. Human Movement Science 28(5), 588-601 (2009)

11. O'Reilly, C., Plamondon, R.: Development of a Sigma-Lognormal Representation for OnLine Signatures. Pattern Recognition 42, 3324-3337 (2009)

12. Plamondon, R.: Strokes against stroke- Stroke for strides. In: 3rd ICFHR, Bari, Italy (September 2012), http://www. icfhr2012. uniba.it/ICFHR2012Invited_I.pdf

13. Di Lecce, V., Dimauro, G., Guerriero, A., Impedovo, S., Pirlo, G., Salzo, A., Sarcinella, L.: Selection of Reference Signatures for Automatic Signature Verification. In: 5th Int. Conf. on Document Analysis and Recognition (ICDAR-5), Bangalore, India, pp. 597-600 (1999)

14. Congedo, G., Dimauro, G., Impedovo, S., Pirlo, G.: A new methodology for the measurement of local stability in dynamical signatures. In: 4th Int. Workshop on Frontiers in Handwriting Recognition (IWFHR-4), Taipei, Taiwan, pp. 135-144 (1994)

15. Impedovo, D., Modugno, R., Pirlo, G., Stasolla, E.: Handwritten Signature Verification by Multiple Reference Sets. In: 11th Int. Conf. on Frontiers in Handwriting Recognition, Concordia University, Montreal, Canada, pp. 125-129 (August 2008)

16. Di Lecce, V., Dimauro, G., Guerriero, A., Impedovo, S., Pirlo, G., Salzo, A.: A Multiexpert System for Dynamic Signature Verification. In: Kittler, J., Roli, F. (eds.) MCS 2000. LNCS, vol. 1857, pp. 320-329. Springer, Heidelberg (2000)

17. Impedovo, D., Pirlo, G.: On-line Signature Verification by Stroke-Dependent Representation Domains. In: 12th Int. Conf. Frontiers in Handwriting Recognition (ICFHR-12), Kolkata, India, pp. 623-627 (November 2010)

18. Yanikoglu, B., Kholmatov, A.: SUSIG: An online handwritten signature database, associated protocol and benchmark results. Pattern Anal. Applic. 12, 227-236 (2009) 